\title{
Effect of atorvastatin on vascular endothelial function in moderately nicotine-dependent smokers
}

\author{
X.Z. Cao, Z.R. Luo, H.M. Zhang and J.Q. Cai \\ Fuzhou General Hospital, Nanjing Command, PLA, Fuzhou, China \\ Corresponding author: J.Q. Cai \\ E-mail: jinquancai@yeah.net
}

Genet. Mol. Res. 13 (2): 2698-2702 (2014)

Received March 20, 2013

Accepted October 4, 2013

Published April 8, 2014

DOI http://dx.doi.org/10.4238/2014.April.8.13

\begin{abstract}
We investigated the effect of atorvastatin on vascular endothelial function in moderately nicotine-dependent smokers. One hundred and sixty moderately nicotine-dependent smokers were randomly divided into the atorvastatin group $(\mathrm{N}=80)$ and the control group $(\mathrm{N}=80)$. Total cholesterol $(\mathrm{TC})$, triglyceride, high-density lipoprotein cholesterol, low-density lipoprotein cholesterol (LDL-C), fasting plasma glucose levels, aspartate aminotransferase, total bilirubin, creatine phosphokinase, and brachial artery flow-mediated vasodilation function (FMD) were measured before and 12 months after atorvastatin treatment. After a 12-month atorvastatin therapy, the TC and LDL-C levels of patients were decreased significantly $(\mathrm{P}<0.05)$ and the FMD of patients were improved significantly ( $\mathrm{P}$ $<0.05)$. Compared with the control group, TC and LDL-C of the patients were significantly decreased $(\mathrm{P}<0.05)$ and the FMD of the patients were significantly improved $(\mathrm{P}<0.05)$. Atorvastatin may significantly improve endothelial function in moderately nicotinedependent smokers.
\end{abstract}

Key words: Atorvastatin; Moderately nicotine-dependence; Vascular endothelial function 


\section{INTRODUCTION}

Coronary heart disease remains the leading cause of death in the United States despite the fact that the death rate related to this disease has decreased $40 \%$ compared to that 30 years ago. Compared with developed countries, the morbidity and mortality of coronary heart disease in China have been increasing and occurring at younger ages. "Early death" caused by cardiovascular disease accounts for $63 \%$ of potential life loss. Smoking is an important risk factor for young and middle-aged patients suffering from coronary heart disease. China has the largest population of smokers worldwide, including up to 350 million people. Smoking severely impairs vascular endothelium (Czernin and Waldherr, 2003), which is the initiating factor leading to cardiovascular and cerebrovascular diseases (de las Heras et al., 1999). Smoking cessation can reduce the risk by $36 \%$ (Critchley and Capewell, 2003). Despite efforts advocating smoking cessation (including medication), the rate of successful smoking cessation in China is less than 3\%. Therefore, it is essential to seek effective drugs against damage to blood vessels by nicotine. Numerous studies have shown that atorvastatin, apart from its lipid-lowering effects, is an antioxidative and anti-inflammatory factor in improving endothelial function (Blum and Shamburek, 2009). Based on this information, we examined the use of atorvastatin and tried to determine whether it can be used to improve vascular endothelial function among smokers and reduce cardiovascular risk.

\section{MATERIAL AND METHODS}

\section{Clinical data}

This study included 160 male moderately nicotine-dependent smokers according to the Fagerstrom test for nicotine dependence (FTND) (Fagerstrom and Schneider, 1989), with an average age of 42.3 years (range, 30-51 years). Individuals who had hypertension, coronary heart disease, diabetes, valvular heart disease, heart failure, connective tissue disease, infection, liver and kidney dysfunction, or bleeding disorders were excluded from the present study. All participants were randomly divided into the atorvastatin group $(\mathrm{N}=80)$ or a control group $(\mathrm{N}=80)$ by using a randomly digital table. Atorvastatin (Lipitor, Pfizer Pharmaceutical Co. Ltd., Dalian, China) was orally administered (20 mg per evening) for 12 months in the atorvastatin group.

\section{Methods}

All participants were given 3-min smoking cessation education by clinicians at each visit, which stressed the danger of smoking on the cardiovascular system, and were advised to quit smoking.

Blood samples were collected at the beginning of the study and 12 months later to measure fasting plasma glucose (FPG), total cholesterol (TC), triglycerides (TG), high-density lipoprotein cholesterol (HDL-C), low-density lipoprotein cholesterol (LDL-C), aspartate aminotransferase (AST), total bilirubin (TBIL), and creatine phosphokinase (CPK) by using an automatic biochemical analyzer. All participants were asked to refrain from alcohol intake, high-fat diet, and extensive exercise $24 \mathrm{~h}$ before blood collection. 
Brachial artery flow-mediated dilation (FMD) was measured by the same investigator who was blinded to clinical data and treatment. After 15 min of lying in a bed, all participants underwent color Doppler ultrasonography and high-frequency linear array ultrasound examination in the dark to measure the basic brachial artery diameter $\left(\mathrm{D}_{0}\right)$, blood flow velocity $\left(\mathrm{V}_{0}\right)$, brachial artery diameter $\left(\mathrm{D}_{1}\right)$, and blood flow velocity $\left(\mathrm{V}_{1}\right)$ after reactive hyperemia according to the methods of Celermajer et al. (1992). The following formula was used to calculate FMD: $\left.\mathrm{FMD}=\left[\left(\mathrm{D}_{1}-\mathrm{D}_{0}\right) / \mathrm{D}_{0}\right)\right] \times 100 \%$.

The nicotine-dependence level of the smokers was assessed 12 months after atorvastatin usage according to the FTND.

\section{Statistical analysis}

Statistical analysis was performed with the SPSS13.0 software. The data are reported as means $\pm \mathrm{SD}$ and the $t$-test was adopted for inter- and intra-group comparisons. $\mathrm{P}<0.05$ was considered to be statistically significant.

\section{RESULTS}

As shown in Table 1, there was no significant difference in age and FTND between the 2 groups $(\mathrm{P}>0.05)$. Differences in TC, TG, HDL-C, LDL-C, FPG, and FMD between groups at the beginning of the study were not statistically significant. All participants were under close observation for an average of 12 months. After atorvastatin treatment, the TC and LDL-C level of the atorvastatin group decreased $(\mathrm{P}<0.05)$, while FMD increased $(\mathrm{P}<$ 0.05). Compared with the control group, the level of TC and LDL-C of the atorvastatin group decreased $(\mathrm{P}<0.05)$ and FMD increased $(\mathrm{P}<0.05)$. Nicotine-dependence level in both groups was reassessed; they remained moderately dependent 12 months later $(\mathrm{P}>0.05)$. No adverse reaction associated with administration of Lipitor was observed. No myocardial infarction, stroke, or death occurred for either group.

\begin{tabular}{|c|c|c|c|c|}
\hline & \multicolumn{2}{|c|}{ Control group $(\mathrm{N}=80)$} & \multicolumn{2}{|c|}{ Atorvastatin group $(\mathrm{N}=80)$} \\
\hline & Before observation & After observation & Before treatment & After treatment \\
\hline Age (years) & \multicolumn{2}{|c|}{$41 \pm 2.1$} & \multicolumn{2}{|c|}{$42 \pm 1.5$} \\
\hline FTND (points) & $6 \pm 0.34$ & $6 \pm 0.56$ & $6 \pm 0.51$ & $6 \pm 078$ \\
\hline $\mathrm{TC}(\mathrm{mM})$ & $5.23 \pm 0.26$ & $5.31 \pm 0.33$ & $5.41 \pm 0.32$ & $4.31 \pm 0.36^{* \#}$ \\
\hline $\mathrm{TG}(\mathrm{mM})$ & $2.27 \pm 0.37$ & $2.12 \pm 0.31$ & $2.33 \pm 0.37$ & $2.25 \pm 0.27$ \\
\hline LDL-C (mM) & $3.11 \pm 0.35$ & $3.18 \pm 0.29$ & $3.15 \pm 0.31$ & $2.14 \pm 0.28^{* \#}$ \\
\hline HDL-C (mM) & $1.89 \pm 0.31$ & $1.95 \pm 0.33$ & $1.98 \pm 0.25$ & $2.01 \pm 0.31$ \\
\hline $\mathrm{FPG}(\mathrm{mM})$ & $5.11 \pm 0.13$ & $5.09 \pm 0.25$ & $5.24 \pm 0.29$ & $5.32 \pm 0.41$ \\
\hline FMD $(\%)$ & $6.81 \pm 0.85$ & $6.12 \pm 0.95$ & $6.63 \pm 0.76$ & $7.95 \pm 1.03^{* \#}$ \\
\hline AST (IU/L) & $31 \pm 0.35$ & $28 \pm 0.71$ & $31 \pm 0.43$ & $30 \pm 0.82$ \\
\hline TBIL $(\mu \mathrm{M})$ & $19 \pm 0.33$ & $20 \pm 0.85$ & $18 \pm 0.78$ & $19 \pm 0.58$ \\
\hline CPK (IU/L) & $139 \pm 0.41$ & $142 \pm 0.25$ & $145 \pm 0.31$ & $138 \pm 0.87$ \\
\hline
\end{tabular}

FTND = fagerstrom test for nicotine dependence; $\mathrm{TC}=$ total cholesterol; $\mathrm{TG}=$ triglycerides LDL-C and HDL-C $=$ low- and high-density lipoprotein cholesterol, respectively; FPG = fasting plasma glucose; FMD = flow-mediated dilation; $\mathrm{AST}=$ aspartate aminotransferase; $\mathrm{TBIL}=$ total bilirubin; $\mathrm{CPK}=$ creatine phosphokinase $. \mathrm{P}<0.05$ *Comparison of atorvastatin group before and after treatment. $\mathrm{P}<0.05{ }^{*}$ Comparison between atorvastatin group (after treatment) and control group (after observation). 


\section{DISCUSSION}

The morbidity and mortality of coronary heart disease in China have been increasing and the ages of those affected are decreasing. Smoking serves as an important risk factor for coronary heart disease, which is particularly true among young and middle-aged patients. Vascular endothelial function disorders (Neunteufl et al., 2002) and endothelial injuries have been detected in these young patients and the occurrence of cardiovascular events is rising (Barua et al., 2002; Czernin and Waldherr, 2003). Therefore, improvement of endothelial function is important for preventing cardiovascular and cerebrovascular diseases.

The widely used FTND is very good for evaluating the effect on nicotine addiction. FTND scoring is significantly correlated with nicotine concentration in blood (Domino, 1998). With a total score of 10 points, a score of 5-6 indicates moderate nicotine dependence (Vink et al., 2005). FTND of 4 or higher suggests a strong possibility of persistent smoking in the future and increased cardiovascular risk (John et al., 2004; Teo et al., 2006). The results of this study suggest that Lipitor reduces TC and LDL-C, while FMD increased among nicotine additive regular smokers with a normal lipid spectrum. FMD is important for evaluating endothelium-dependent vasodilatation function (Jambrik et al., 2004), reflecting coronary artery endothelial function (Jambrik et al., 2004), as well as an important indicator of cardiovascular risk factors and cardiovascular accidents (Muiesan et al., 2008). Therefore, the present study suggested that Lipitor can improve endothelial function among regular smokers of substances with nicotine additives. The proposed mechanisms are as follows: 1) Lipitor can regulate lipoprotein intake by vascular endothelial cells, regulate cell cholesterol metabolism, reduce plasma endothelin level, increase calcitonin gene-related peptide level, and protect the endothelial function steadily (Batova et al., 2006). 2) It can reduce LDL oxidation and proliferation of smooth muscle cells (Mason et al., 2008). 3) It can directly activate NO synthetase, cause rapid release of NO in endothelial cells, and lead to endothelium-dependent vasodilatation (Meda et al., 2010). 4) Lipitor can upregulate endothelial NO synthase mRNA expression by post-transcriptional control and improve activity of NO synthase, and hydroxymethylglutaryl coenzyme A reductase inhibitor can completely prevent Ox-LDL in its downregulation of NO synthase (Laufs et al., 1998). During the average 12-month follow-up visit, cardiovascular occurrence was not observed in either group, which may be related to the short-observation period. Therefore, atorvastatin is thought to improve endothelial function among nicotine additive regular smokers. Further studies over a longer period will be required to determine whether atorvastatin can reduce the risk of cardiovascular diseases in the future.

\section{REFERENCES}

Barua RS, Ambrose JA, Eales-Reynolds LJ, DeVoe MC, et al. (2002). Heavy and light cigarette smokers have similar dysfunction of endothelial vasoregulatory activity: an in vivo and in vitro correlation. J. Am. Coll. Cardiol. 39: 1758-1763.

Batova S, DeWever J, Godfraind T, Balligand JL, et al. (2006). The calcium channel blocker amlodipine promotes the unclamping of eNOS from caveolin in endothelial cells. Cardiovasc. Res. 71: 478-485.

Blum A and Shamburek R (2009). The pleiotropic effects of statins on endothelial function, vascular inflammation, immunomodulation and thrombogenesis. Atherosclerosis 203: 325-330.

Celermajer DS, Sorensen KE, Gooch VM, Spiegelhalter DJ, et al. (1992). Non-invasive detection of endothelial dysfunction in children and adults at risk of atherosclerosis. Lancet 340: 1111-1115.

Critchley JA and Capewell S (2003). Mortality risk reduction associated with smoking cessation in patients with coronary heart disease: a systematic review. JAMA 290: 86-97. 
Czernin J and Waldherr C (2003). Cigarette smoking and coronary blood flow. Prog. Cardiovasc. Dis. 45: 395-404.

de las Heras N, Aragoncillo P, Maeso R, Vazquez-Perez S, et al. (1999). AT(1) receptor antagonism reduces endothelial dysfunction and intimal thickening in atherosclerotic rabbits. Hypertension 34: 969-975.

Domino EF (1998). Tobacco smoking and nicotine neuropsychopharmacology: some future research directions. Neuropsychopharmacology 18: 456-468.

Fagerstrom KO and Schneider NG (1989). Measuring nicotine dependence: a review of the Fagerstrom tolerance questionnaire. J. Behav. Med. 12: 159-182.

Jambrik Z, Venneri L, Varga A, Rigo F, et al. (2004). Peripheral vascular endothelial function testing for the diagnosis of coronary artery disease. Am. Heart J. 148: 684-689.

John U, Meyer C, Hapke U, Rumpf HJ, et al. (2004). Nicotine dependence, quit attempts, and quitting among smokers in a regional population sample from a country with a high prevalence of tobacco smoking. Prev. Med. 38: 350-358.

Laufs U, La Fata V, Plutzky J and Liao JK (1998). Upregulation of endothelial nitric oxide synthase by HMG CoA reductase inhibitors. Circulation 97: 1129-1135.

Mason RP, Kubant R, Heeba G, Jacob RF, et al. (2008). Synergistic effect of amlodipine and atorvastatin in reversing LDL-induced endothelial dysfunction. Pharm. Res. 25: 1798-1806.

Meda C, Plank C, Mykhaylyk O, Schmidt K, et al. (2010). Effects of statins on nitric oxide/cGMP signaling in human umbilical vein endothelial cells. Pharmacol. Rep. 62: 100-112.

Muiesan ML, Salvetti M, Paini A, Monteduro C, et al. (2008). Prognostic role of flow-mediated dilatation of the brachial artery in hypertensive patients. J. Hypertens. 26: 1612-1618.

Neunteufl T, Heher S, Kostner K, Mitulovic G, et al. (2002). Contribution of nicotine to acute endothelial dysfunction in long-term smokers. J. Am. Coll. Cardiol. 39: 251-256.

Teo KK, Ounpuu S, Hawken S, Pandey MR, et al. (2006). Tobacco use and risk of myocardial infarction in 52 countries in the INTERHEART study: a case-control study. Lancet 368: 647-658.

Vink JM, Willemsen G, Beem AL and Boomsma DI (2005). The fagerstrom test for nicotine dependence in a dutch sample of daily smokers and ex-smokers. Addict. Behav. 30: 575-579. 